\title{
The Effects of Community Openness on the Passage of Surrounding Roads
}

\author{
Nijie Chao ${ }^{1, a}$ \\ ${ }^{1}$ North China Electric Power University (Baoding), Baoding 071000, China; \\ a1363661344@qq.com
}

Keywords: open community, traffic flow, traffic capacity, retardant increase model

\begin{abstract}
In model 1, by assuming different delayed time, we obtain the effects of road passage capability under different delayed time, and draws out the single lane passage capability and the road passage capability curve under different delay (taking the community with the unilateral length of 300 meters as the example), it is found that the speed decrease is not obvious in the primary road interval, and obtains the conclusion that the road passage capability is not weakened nearly.

In model 2, we consider the effects of intersection on the upstream road intersection in the situation that the congestion has occurred, which is also the situation that should be avoided in the road design. Therefore, we introduce the idea of vehicle queuing phenomenon. According to the queuing length increasing rate, we build the retardant increase model (namely model two) to obtain the relations between queuing length and time.
\end{abstract}

\section{Introduction}

This paper mainly studies the problem whether the open community can improve the road passage capability and improve the traffic situation. We mainly apply the traffic low thinking, queuing theory to obtain the evaluation system with the main evaluation indexes of passage capability and time allowance. On the basis, considering the delay of intersection on the traffic flow interval speed, and the effects of intersection on the upstream intersection, the passage capability model and the retardant increase model based on queuing theory is obtained.

\section{Traffic capability model}

\section{Traffic open community area definition.}

In the domestic Urban Road Traffic Planning Design Standards, the branch road network density is $3-5 \mathrm{~km} / \mathrm{km}^{2}$,the urban primary road network and secondary primary road density is $0.8-1.2$ $\mathrm{km} / \mathrm{km}^{2} 、 1.2-1.4 \mathrm{~km} / \mathrm{km}^{2}$, the traffic main road interval of the city is generally $700-1200 \mathrm{~m}$.【1】

\section{Single lane basic running capability.}

The so-called road traffic capability refers to the maximum traffic volume in some lane or some fracture surface in the unit time (amortized to the equivalent standard vehicle amount).【2】

The calculation formula of single lane basic traffic capability is as below:

$$
N_{0}=\frac{3600}{I / v}=\frac{3600}{I / \frac{V}{3.6}}=\frac{1000 \mathrm{~V}}{I}
$$

In the equation, $\mathrm{N}_{0}$ is the basic passage capability (pcu/h) ; l is the head interval (m); $\mathrm{V}$ is the running speed $(\mathrm{km} / \mathrm{h})$, and

$$
L=I_{\text {车 }}+\frac{V}{3.6} \times \mathrm{t}+\frac{\mathrm{K}_{2}-K_{1}}{254(\psi+\mathrm{f})} \times V^{2}+I_{\text {安 }}
$$

In the equation, $l_{\text {车 }}$ is the vehicle length, this paper takes the average value as 5 meters and $\mathrm{V}$ as the running speed. We believe that the speed limit in the downtown is 30 kilometers per hour. $t$ is the response time of the driver. $K_{2}$ is the braking security coefficient of the behind vehicle, $K_{1}$ is the braking security coefficient of the front vehicle, according to the former Soviet Union's load heavy vehicle, $K_{2}-K_{1}=0.67$ is derived. $\Psi$ is the adhesive coefficient between tire and the road, usually 0.2-0.5, and this paper adopts 0.35 .f is the sliding resistance coefficient, usually 0.02 。 $1_{\text {安 }}$ is the security distance between vehicles $(\mathrm{m})$, usually 2-5 (m), and this paper adopts $2(\mathrm{~m})$. 


\section{The passage capability under intersection delay.}

According to the assumption of the above paper, we believe that for the consideration of security, the vehicles passing the intersection will make the move of slowing down, the time is increased and the interval speed is decreased. We assume that the process of slowing down before speeding up will increase 4 seconds, using $\mathrm{D}$ to express the delayed times.

Taking the community with $\mathrm{L}=300$, or the unilateral length of 300 meters as the example.

After the delay, the relations between interval speed V'and V. Substitute V and V' into the formula of single lane basic passage capability $\mathrm{N}_{0}$, other parameters are substituted as the above, simplified to obtain the following equation:

$$
N_{0}=\frac{1000 \mathrm{~V}}{0.0071 V^{2}+0.347 V+7} \quad N_{0}=\frac{1000 V^{\prime}}{0.0071 V^{\prime 2}+0.347 V^{\prime}+7}
$$

For the convenience of observation, we will draw the effects of road passage abilities $\mathrm{N}_{0}$ under different delayed time $(D=1 、 D=2)$ as below:

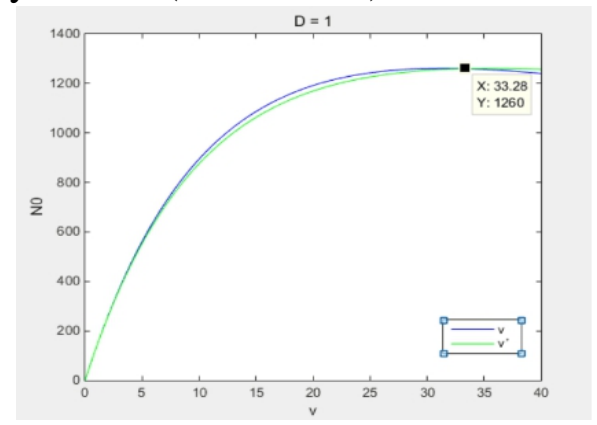

Figure 1

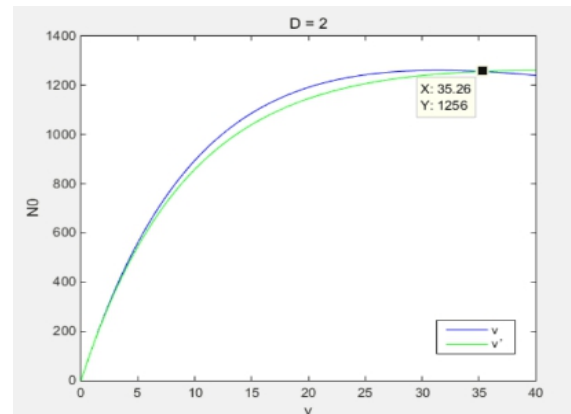

Figure 2

In the figure, the vertical axis is the passage capability $\mathrm{N}_{0}(\mathrm{pcu} / \mathrm{h})$, the horizontal axis is the averaged interval speed $(\mathrm{km} / \mathrm{h})$.

\section{Queuing model}

\section{Queuing phenomenon.}

According the research by Bing Yu 【4】, the production of queuing phenomenon is because the actual passage vehicle in the unit time is smaller than the vehicle driven from the upstream section, with the accumulation of time, the vehicle queuing phenomenon is caused by the original cross section.

Retardant increase model.

With the increase of vehicle queuing length, the retardant function has been greater; the retardant function is also shown on the effects of queuing length increasing rate $r$, the stronger the retardant function, the lower the increasing rate will be. Therefore, if $r$ is expressed the function $r(y)$ of the queuing length $y$, it should be the decreasing function of y. Namely:

$$
\frac{d y}{d t}=n_{(y)} \times y \quad y_{(0)}=y_{0}
$$

Assuming $\mathrm{r}(\mathrm{y})$ is the linear function of $\mathrm{y}$, namely:

$$
r_{(y)}=r_{0}-s \times y \quad(s r>0)
$$

In the equation: $r_{0}$ represents the increasing rate of vehicle queuing length when the congestion starts, called as the original increasing rate.

In order to solve the coefficient $\mathrm{s}$, we need to introduce the longest distance $\mathrm{y}_{\mathrm{m}}$ from the congestion occurring place to the upstream intersection. Obviously, when $\mathrm{y}_{\mathrm{y}} \mathrm{y}_{\mathrm{m}}$, the vehicle queuing length reaches the extreme, and the queuing length increasing rate $\mathrm{r}\left(\mathrm{y}_{\mathrm{m}}\right)=0$ at this time. Substitute

it into the above equation, obtaining:

$$
\left\{\begin{array}{c}
\frac{d y}{d t}=r_{(y)} \times y=r_{0} \times y \times\left(1-\frac{y}{y_{m}}\right) \\
y_{(0)}=y_{0}
\end{array}\right.
$$

As the above equation, it can directly see that roy factor shows the increasing trend of vehicle 
queuing length,(1-y/ym) factor shows the retardant function of the length between the congestion occurring place to the upstream intersection road on the increase of queuing length. MATLAB is used to solve the above to obtain the expression equation of queuing length $\mathrm{y}$ on the time $\mathrm{t}$ :

$$
y=\frac{y_{m}}{1+\left(\frac{y_{m}}{y_{0}}-1\right) \times e^{-r}}
$$

In the following, we take the community with the unilateral length of 400 meters as the example, assuming that a traffic intersection is opened at the central point of one side in this area. Obviously, when vehicle traffic flow increases suddenly, and the congestion occurs in the intersection, the maximum queuing distance from the congestion point to the upstream crossed intersection $\mathrm{y}_{\mathrm{m}}=200$ meters.. While our goal is to propose the advice to urban planning and traffic administration department, we take the relatively rigorous parameters to obtain the general conclusion.

Here, we take original increasing rate $\mathrm{r}_{0}=0.4,0.6$ or 0.8 (pcu/min) separately and the original queuing length of 15 meters to obtain the functional curve of queuing length on time as below figure 3:

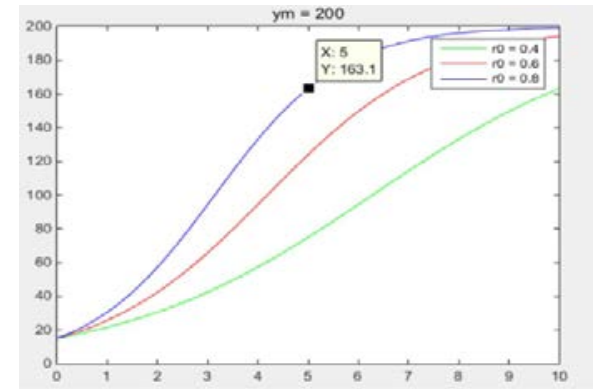

Figure 3

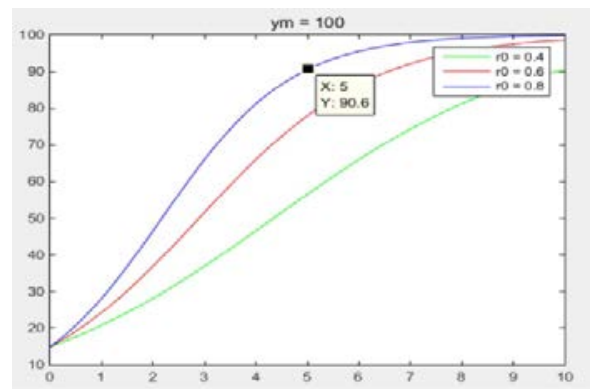

Figure 4

As the figure, if we believe that the congestion evacuation abilities of the block is five minutes, it can be shown that even if in the situation of larger queuing length with the original increasing rate, the intersection of the open community will not cause effects on the upstream intersection.

In the following, in order to roughly estimate the unilateral length of the community to be opened, we will take a community with the unilateral length of 200 meters, still take the original increasing rate $\mathrm{r}_{0}=0.4$ or 0.6 or $0.8(\mathrm{pcu} / \mathrm{min})$, and the original queuing length of 15 meters. Differently, the opened cross intersection has only 100 meters distance from the upstream intersection at the central point of this primary road, namely the maximum queuing length $\mathrm{y}_{\mathrm{m}}=100$ meters.

At this time, the functional curve of the queuing length on time is as above figure 4.

From the figure, it can be seen that when the original increase rate is higher, the congestion of this open community's intersection has nearly spread to the upstream intersection within five minutes, which causes the regional blocking.

\section{Conclusions}

First of all, taking the community with the unilateral length of 300 meters, one and even two community intersections are placed aside. Although it triggers the lower sectional speed in the main primary road, and the decrease of smooth level, it has not reduced the passage abilities of the road, and the curves of the above two figures demonstrate this point.

Besides, the community opening intersection position and the density selection will directly affect the blocking possibility in the surrounding sections. If the distance between neighboring cross roads in the open community is shorter or the distance to the upstream intersection is shorter, once the congestion occurs in the opening community intersection, it will affect the upstream intersection very soon. According to the parameters set in this paper, we can believe that the community with the unilateral length less than 200 meters is not suitable to open. 


\section{References}

[1] Zhiyong Cao. New Urbanism Theory—New Perspective of Chinese Urban Design[M]. Nanjing: Southeast University,2011.

[2] Hong Zhu, Jingjie Zhang, Zhenzhao Liang, Yaxuan Zhang.The Analysis and Forecast of the Effects of Lane Occupation on Road Traffic Passage capability Based on M/M/I/K Model[J]. Practice and Understanding of Mathematics,2015,45(2),225-231.

[3] Fengyao Zhou, Lingli Bu, etc. Research Current Situation of Urban Road Traffic Abilities and its Development Trend[J] Road and Automobile Transportation, 2013,1(1),32-35.

[4] Bing Yu, Jian Lu, Xiaoya Tao. Influential Range Algorithm of Road Traffic Accidents[J].Urban Traffic, 2008,6(3),82-86. 